\title{
Histologic variants of urothelial bladder cancer and nonurothelial histology in bladder cancer
}

\author{
Venu Chalasani, MD; Joseph L. Chin, MD, FRCSC; Jonathan I. Izawa, MD, FRCSC
}

\begin{abstract}
Bladder cancer can be classified histologically as urothelial or non-urothelial. Urothelial cancer has a propensity for divergent differentiation, which has increasingly been recognized in recent years due to heightened awareness and improved immunohistochemistry techniques. Furthermore, the recent World Health Organization classification of urothelial cancers improved clarity on this issue, with its listing of 13 histologic variants of urothelial cancer. The divergent differentiation patterns include, amongst others, squamous, glandular, micropapillary, nested, lymphepithelioma-like, plasmacytoid and sarcomatoid variants of urothelial cancer. Attempts to quantify the amount of divergent differentiation present, such as using the nonconventional differentiation number, have been made recently, which will improve the ability to compare publications from different centres. Genetic-based studies have indicated that the histologic variants of urothelial cancer arise from a common clonal precursor. Mostly, the current evidence suggests that urothelial cancer with divergent differentiation has a worse prognosis when compared with pure urothelial cancer. This article will review the current literature on variant histologies of urothelial cancer, and well as new developments in pure squamous cell carcinoma, small cell carcinoma and adenocarcinoma of the bladder.
\end{abstract}

Can Urol Assoc J 2009;3(Suppl4):S193-8

\section{Introduction}

The spectrum of bladder cancer is quite diverse, with urothelial cancer making up the vast majority. ${ }^{1,2}$ Bladder cancer is the sixth most common cancer in Canada, and the ninth leading cause of cancer mortality, with 1800 deaths estimated in 2008. ${ }^{3}$ Urothelial cancer, which represents more than $90 \%$ of bladder cancers, is mostly found in its pure form. However, urothelial cancer is known to show variant histologic features, otherwise known as divergent differentiation (Table 1), ${ }^{4}$ with estimates ranging from $7 \%$ to $81 \%$ in series specifically reporting the differentiation patterns of urothelial cancer. ${ }^{5-7}$ The large variation in the reported incidence of divergent differentiation (mixed histologic features) is likely due to differences in sampling techniques and improved accuracy with recognition in recent years of the possibility that outcomes may be related to histologic subtypes present within the specimen, even in small areas. ${ }^{7}$ Kassouf and colleagues analyzed the predictors of survival for a cohort of patients with node-positive urothelial cancer who had undergone neoadjuvant chemotherapy. The authors found that on multivariate analysis histologic type (pure urothelial versus urothelial with mixed histologic features) remained a significant independent predictor of overall survival as well as recurrence-free survival. ${ }^{8}$

In recent years, attempts have been made to quantify the amount of divergent differentiation seen within a urothelial carcinoma. ${ }^{5,7}$ Does divergent differentiation within a urothelial bladder cancer affect outcomes? This is the key question for clinicians and the focus of this review. Patients with non-urothelial primary bladder cancer, such as pure squamous cell carcinoma, pure bladder sarcoma or pure adenocarcinoma, are known to have different outcomes, 9,10 and hence different management, when compared to urothelial carcinoma. It is unclear whether this result extends to urothelial cancer patients with divergent differentiation. Until recently, the body of opinion has been that squamous or glandular divergent differentiation are not clinically important as the prognosis is not affected. ${ }^{11,12}$ This article will review the current literature on variant histologies of urothelial cancer and new developments in pure squamous cell carcinoma, small cell carcinoma and adenocarcinoma of the bladder.

\section{Variant urothelial histology noted at transurethral resection}

In a study of 165 transurethral resections, Billis and colleagues noted that $7 \%$ of patients showed squamous or glandular differentiation. ${ }^{6}$ These patients had statistically significant higher clinical stage at presentation, with none of the patients having superficial disease at presentation. A larger study by Wasco and colleagues, of 448 transurethral resections, found that $25 \%$ of patients had divergent differentiation, of which all were high grade, and all but one were invasive at presentation. ${ }^{13}$ The histologic subtypes noted were squamous, glandular, sarcomatoid, micropapillary, small cell and plasmacytoid. After excluding all low-grade urothelial cancers, Wasco and colleagues noted that urothelial cancers with 
Chalasani et al.

Table 1: Variants of invasive urothelial carcinoma

\begin{tabular}{l} 
Squamous differentiation \\
Glandular differentiation \\
Nested pattern \\
Microcystic \\
Micropapillary \\
Lymphoepithelioma-like \\
Plasmacytoid and lymphoma-like \\
Sarcomatoid/carcinosarcoma \\
Giant cell \\
Trophoblastic differentiation \\
Clear cell \\
Lipid cell \\
Undifferentiated \\
\hline Adapted from the World Health Organization Classification of Tumours. Pathology and \\
Genetics of Tumours of the Urinary System and Male Genital Organs. ${ }^{4}$
\end{tabular}

divergent differentiation were more likely to be invasive $(p<0.001)$ when compared to pure urothelial cancers. Furthermore, on multivariate logistic modelling, the presence of divergent differentiation was an independent predictor of extravesical tumour extension on the final radical cystectomy specimen. Based on these studies, clinicians faced with mixed histologic features on a transurethral resection report should maintain a high index of suspicion for the presence of a more aggressive variant of bladder cancer with a higher likelihood of extravesical disease.

\section{Quantifying the amount of divergent differentiation present within the specimen}

Unlike non-seminomatous testicular cancer, where it is commonplace to quantify the amount of each histologic subtype present within the tumour, there is currently no standard reporting methodology with regards to the amount of variant histology (i.e., divergent differentiation) present within urothelial cancer. Furthermore, the clinical significance would be unclear, even if the differing components were quantified. Jozwicki and colleagues performed a mapping study in 38 cystectomy specimens, and then correlated the mapping studies with survival time. They suggested that the presence of greater than $80 \%$ pure urothelial cancer within a specimen was a favourable prognostic factor, and that increasing numbers of histologic subtypes (i.e., increasingly divergent differentiation) led to a less favourable prognosis.

In a follow-up to this study, Domanowska and colleagues correlated the number of histologic variants present with lymph node metastasis. ${ }^{7}$ They analyzed 101 cystectomy specimens and used a threshold for diagnostic importance of $6.5 \%$ for histologic variants. They have suggested counting the number of all diagnostically important histologic patterns within the tumour, and then recording that as the nonconventional differentiation number (NDN). On multivariate regression models, only NDN remained an inde- pendent predictor for positive lymph nodes. This study highlights the need for the development of a standardized pathologic reporting system for histologic variants of urothelial cancer to compare results across studies.

\section{Squamous differentiation}

Squamous differentiation is the most common variant of urothelial cancer, with recent reports noting its presence in $16.8 \%$ to $22.1 \%$ of cases. ${ }^{14-16}$ It is characterized by the presence of keratinization or intercellular bridges. ${ }^{1}$ Antunes and colleagues analyzed the outcomes of 113 patients treated with radical cystectomy. They found squamous differentiation (present in $22.1 \%$ of cases) was an independent predictor of cancer specific survival on multivariate analysis $(p=0.002)$. Similar findings were reported by Erdemir and colleagues. They noted higher recurrence and progression rates. ${ }^{17}$ Honma and colleagues, in a study of 27 patients with local recurrence after radical cystectomy, found that any element of squamous differentiation was the only independent predictor of local recurrence in multivariate analysis. ${ }^{18}$ In contrast, Lopez-Beltran and colleagues found the presence of squamous differentiation to have no effect on survival; they did note, however, that it was correlated with higher grade and stage. ${ }^{16}$ Currently, larger studies are needed to ascertain whether or not, on a stage-by-stage basis, patients with squamous differentiation have a worse prognosis than patients with pure urothelial carcinoma. Subsequent to that, it remains to be defined whether or not these patients need different management protocols, as there have been suggestions in the literature of poor responses to chemotherapy and radiotherapy. ${ }^{19,20}$

\section{Glandular differentiation}

Glandular differentiation is an infrequent variant of urothelial cancer, with an incidence of up to $16 \% .^{7}$ Patients with only glandular differentiation should be labelled as adenocarcinoma, rather than urothelial cancer with glandular differentiation. Most published reports have not looked at glandular differentiation in isolation, but rather have combined it with squamous differentiation. Due to the paucity of literature on this variant, the issue of poorer outcomes with this variant has not been resolved, and no concrete recommendations can be made.

\section{Nested variant}

This is an aggressive variant of urothelial cancer which is rare and may sometimes be confused with prominent von Brunn's nests or cystitis cystic. ${ }^{12}$ The limited data in the literature suggests a poor outcome for this group of patients. ${ }^{21,22}$ 


\section{Micropapillary carcinoma}

This variant of urothelial cancer, with a reported incidence as high as $6 \%,{ }^{23}$ has histologic similarity to papillary serous carcinoma of the ovary. ${ }^{24}$ Initial case series showed the advanced stage $^{25}$ and poor prognosis for this variant of urothelial carcinoma, ${ }^{26}$ especially when it was present in high proportions. ${ }^{23,27}$ The largest case series, consisting of 100 consecutive patients with micropapillary variant, reported 5 -year and 10-year Kaplan-Meier overall survival rates of $54 \%$ and $27 \%$, respectively. ${ }^{28}$ Of note, the authors of this series found that patients with this variant (in the noninvasive setting) had poor responses to intravesical therapy, and recommended that patients be offered immediate radical cystectomy. ${ }^{28,29}$

\section{Lymphepithelioma-like carcinoma}

This rare variant of urothelial carcinoma has been described mostly in small case series, ${ }^{30}$ with the largest series having 28 cases. ${ }^{31}$ Most reports, consisting of either single cases or very small series, had suggested a favourable prognosis if lymphepithelioma-like carcinoma was the predominant pattern, as chemotherapy seemed to produce responses. ${ }^{32-34}$ However, the largest series of 28 cases from Tamas and colleagues failed to show an improved prognosis for this variant of urothelial carcinoma. They noted a similar prognosis when compared with patients with conventional urothelial carcinoma who underwent cystectomy. ${ }^{31}$ Currently there is insufficient evidence in the literature to guide therapy, as both surgery and chemotherapy appear to produce responses in anecdotal literature reports. ${ }^{35}$

\section{Plasmacytoid}

Plasmacytoid urothelial carcinoma is a rare variant, with small case series described in the literature, none of which none have more than 10 patients. ${ }^{36,37}$ Although there is insufficient evidence to make any concrete recommendations from these small case series, this variant has displayed aggressive behaviour in the cases described thus far.

\section{Sarcomatoid carcinoma with/without heterologous elements (carcinosarcoma)}

This rare biphasic variant of urothelial carcinoma has, until recently, only been described in small case series, which have suggested a poor outcome for patients with this variant. ${ }^{38}$ Genetic studies suggest that the epithelial and sarcomatoid elements share a common monoclonal cell origin. 39,40 The reported incidence has ranged from $0.2 \%$ to $4.3 \% .{ }^{41}$ This suggestion has been confirmed in an analysis of the Surveillance, Epidemiology and End Results (SEER) database. In total, 135 cases of sarcomatoid and 166 cases of carcinosarcoma were identified, from a total of 182283 patients with primary bladder cancer. ${ }^{42}$ Black and colleagues confirmed that patients have worse disease-specific and overall survival, even after adjusting for stage, when compared to patients with high-grade pure urothelial cancer. No published articles have focused on treatment for this group of patients, although one group has suggested that radical cystectomy be the preferred option for patients with T1 disease, rather than intravesical therapy. ${ }^{41,43}$

\section{Neoadjuvant chemotherapy for urothelial cancer with divergent differentiation}

There have been no prospective randomized trials specifically addressing the role of neoadjuvant chemotherapy prior to radical cystectomy for patients with divergent differentiation. The largest randomized trial of neoadjuvant chemotherapy did not exclude patients with divergent differentiation; however, it did not report the subgroup analysis for this group of patients. ${ }^{44}$ In the absence of data, it would seem reasonable to treat patients with urothelial cancer and squamous or glandular differentiation in the same manner as patients without divergent differentiation, ${ }^{43}$ with the background knowledge that traditionally pure squamous carcinomas are not as responsive to chemotherapy as pure urothelial cancer. ${ }^{45}$ For cases of urothelial cancer with sarcomatoid or micropapillary differentiation, there is no good published evidence, although some authors have suggested that the effect of neoadjuvant cheomotherapy is low, and hence have recommended immediate cystectomy. ${ }^{46}$

\section{Squamous cell carcinoma}

Pure squamous cell carcinoma of the bladder is an uncommon cause of bladder cancer in the developed world, accounting for $2.7 \%$ of bladder cancers in recent series. ${ }^{47}$ However, it is the most common cause of bladder cancer in areas where schistosomiasis is endemic, where it accounts for up to $59 \%$ of bladder cancers. ${ }^{48}$ Risk factors for pure squamous cell carcinoma include chronic inflammation (e.g., long term catheters or bladder calculi), recurrent urinary tract infections, schistosomiasis and prior exposure to cyclophosphamide chemotherapy. ${ }^{49}$

Squamous cell carcinoma often presents at an advanced stage, and, until recently, it was unclear whether patients had a worse prognosis on a stage-for-stage basis when compared with patients with urothelial cancer. ${ }^{47}$ However, a recent study of the SEER database by Scosyrev and colleagues has shown that squamous cell carcinoma was more aggressive than urothelial cancer after adjusting for common prognostic factors, such as stage. ${ }^{50}$ The authors looked at 1422 squamous cell cancers and used 107613 urothelial cancers 
as the comparison group from the SEER database. After adjusting for demographic factors, stage, grade, and initial treatment regimen, squamous cell cancer was an independent predictor of mortality amongst patients with American Joint Committee on Cancer (AJCC) stage III and IV disease, and amongst patients with AJCC stage I and II disease who did not undergo cystectomy as part of their initial treatment regimen.

Surgery is the cornerstone of treatment for this group of patients. Urologists should be aware of the high likelihood of local failure as the leading presentation of recurrence. 9,41,49 Recent series based on the nonbilharzial squamous cell cancer population have shown 2-year recurrence-free survival rates of $32.8 \%,{ }^{9}$ and 5 -year recurrence-free survival rates of $43 \% .{ }^{51}$ To address the issue of local failure after cystectomy for mostly squamous cell carcinoma patients, Zaghloul and colleagues randomized 236 patients to adjuvant radiotherapy vs. observation. There is an absolute risk reduction of $19 \%$ ( $44 \%$ vs. $25 \%$ ) shown in this trial of cystectomy plus adjuvant radiotherapy versus cystectomy alone. ${ }^{52}$ Current chemotherapy regimens are not satisfactory, with further randomized trials needed in this area ${ }^{45}$. However, in the neoadjuvant setting there has been 1 randomized trial, which compared 2 courses of epirubicin preoperatively plus cystectomy and 4 courses of epirubicin postoperatively versus cystectomy alone. ${ }^{53}$ This randomized study of 71 patients demonstrated disease-free survival rates of $73.5 \%$ (epirubicin and cystectomy group) versus $37.9 \%$ (cystectomy alone group) at 2 years. Long-term data from this study has not been published. There has also been 1 published abstract of a randomized trial of neoadjuvant cisplatin and gemcitabine involving 120 patients, which showed no benefit. ${ }^{54}$ It must be borne in mind that both of these trials were conducted in patients with squamous cell cancer secondary to the bilharzial bladder, and therefore may not be representative of the patients in the developed world. Hence, there is inconclusive evidence to recommend routine neoadjuvant chemotherapy for squamous cell cancer of the bladder, and further trials are needed.

\section{Adenocarcinoma}

Primary adenocarcinoma of the bladder is rare in the developed world, accounting for about $1.4 \%$ of bladder cancers undergoing radical cystectomy; 47,55 it is more prevalent in the developing world, accounting for up to $11 \%{ }^{48}$ It is the third most common bladder cancer, after urothelial cancer and squamous cell cancer. ${ }^{1}$ Primary adenocarcinoma of the bladder can be histologically classified as enteric, adenocarcinoma not otherwise specified, signet ring cell, mucinous, clear cell, hepatoid or mixed. ${ }^{4}$ Recent publications have suggested that the extent of signet ring cell expression within the adenocarcinoma may be associated with wors- ening survival. ${ }^{56}$ Furthermore, it also often encompasses urachal cancer, which, while most commonly an adenocarcincoma, can be squamous or urothelial in origin. ${ }^{4}$ Results from the SEER database show that urachal cancer makes up about $10 \%$ of all adenocarcinomas of the bladder. ${ }^{57}$

In general, most published series have shown a poor prognosis for adenocarcinoma of the bladder, although it is unclear if this is due to advanced stage at presentation. Wright and colleagues, in an analysis of the SEER database, found the 5 -year survival of 151 patients with urachal cancer was 48\%, while it was $35 \%$ for 1374 patients with non-urachal adenocarcinoma of the bladder. ${ }^{57}$ Series from Egypt, where the prevalence of adenocarcinoma of the bladder is higher, have shown 5 -year disease-free survival rates of $46 \%$ to $55 \%$. ${ }^{58,59}$ Local recurrence has been reported to be the most common cause of treatment failure in a series of 192 patients by Zaghloul and colleagues. ${ }^{59}$ The same authors also noted higher survival in the group that underwent postoperative adjuvant radiotherapy, although it was not a randomized trial.

Most patients with urachal cancer are treated with partial cystectomy and resection of the urachal ligaments and umbilicus, ${ }^{57}$ while patients with non-urachal adenocarcinoma are treated with radical cystectomy. ${ }^{57}$ There have been no prospective randomized trials using radiotherapy or chemotherapy in this area. There is currently a phase II trial that has shown promising preliminary results using 5fluorouracil based chemotherapy instead of the standard urothelial chemotherapeutic approach. ${ }^{60}$

\section{Small cell carcinoma}

Small cell carcinoma is slightly unusual with regards to its histological classification in the latest recommendations from the World Health Organization. Any amount of small cell histology, even when present with predominantly urothelial elements, warrants the classification of primary small cell bladder cancer, rather than the classification of urothelial carcinoma with small cell differentiation. ${ }^{4}$ This is largely due to the fact that the prognosis of the patient is determined by the small cell histology, even when present in small amounts in a tumour that is primarily urothelial in origin. Non-small cell elements, commonly urothelial or glandular, are often present. ${ }^{61}$ Small cell carcinoma is a rare primary tumour of the bladder, accounting for about $1 \%$ of cases undergoing cystectomy. ${ }^{47}$ The key to management is the realization that small cell carcinoma of the bladder is a systemic disease, and, as such, chemotherapy is crucial to achieving good outcomes. ${ }^{62}$

Most published evidence consists of small case series. ${ }^{61,63-65}$ Given the fact that small cell carcinoma is predominantly a systemic disease, some authors have argued that radical cystectomy for local control is not ideal. Bex and colleagues recently published a series of 17 patients treated with 
chemoradiation, for whom the median survival was 32.5 months. This report is similar to published reports with cystectomy; however, the authors only treated patients with limited disease with chemoradiation. ${ }^{64}$ Most published reports are based on cystectomy rather than radiotherapy. Mackey and colleagues, in a series of 106 patients, found that the only independent predictors of improved survival on multivariate analysis were radical surgery and cisplatin chemotherapy. ${ }^{66}$ However, without a randomized trial, it will remain unclear whether this group of patients should be treated with radical cystectomy or radiotherapy for local control. Based on the limited evidence available, it would appear reasonable for patients to be treated with chemotherapy (using a neuroendocrine regimen) followed by radical cystectomy. ${ }^{41}$

\section{Conclusion}

Increasing attention in recent years, along with more specific and sensitive markers, has led to heightened recognition of the divergent differentiation often present in urothelial cancer. The most commonly expressed variants are squamous, glandular, sarcomatoid and micropapillary. The most common non-urothelial histologies in bladder cancer are squamous, adenocarcinoma and small cell carcinoma, all of which often present at an advanced stage. Further prospective studies are needed to accurately clarify the prognosis for these patients, and to develop tailored treatment paradigms. Until then, urologists must maintain a high index of clinical suspicion for aggressive disease in patients who present with either urothelial cancer with divergent differentiation or non-urothelial histology.

From the Departments of Surgery \& Oncology, Divisions of Urology \& Surgical Oncology, London Health Sciences Centre-Victoria Hospital, London, ON

Competing interests: None declared.

This paper has been peer-reviewed.

\section{References}

1. Petersen RO, Sesterhenn I, Davis CJ. Urologic pathology. $3^{\text {rd }}$ ed. Philadelphia, PA: Lippincott Williams \& Wilkins; 2009:636.

2. Mikuz G. Clinical pathology of urologic tumours. Boca Raton, FL: CRC Press; 2007:253.

3. Canadian Cancer Society/National Cancer Institute of Canada. Canadian Cancer Statistics 2008. Toronto, Canada; 2008

4. Eble JN, Sauter G, Epstein Jl, et al. eds. World Health Organization Classification of Tumours. Pathology and Genetics of Tumours of the Urinary System and Male Genital Organs. IARC Press: Lyon;2004:359.

5. Jozwicki W, Domaniewski J, Skok Z, et al. Usefulness of histologic homogeneity estimation of muscleinvasive urinary bladder cancer in an individual prognosis: A mapping study. Urology 2005;66:1122-6.

6. Billis A, Schenka AA, Ramos CC, et al. Squamous and/or glandular differentiation in urothelial carcinoma: Prevalence and significance in transurethral resections of the bladder. Int Urol Nephrol 2001;33:631-3.

7. Domanowska E, Jozwicki W, Domaniewski J, et al. Muscle-invasive urothelial cell carcinoma of the human bladder: Multidirectional differentiation and ability to metastasize. Hum Pathol 2007;38:741-6.
8. Kassouf W, Agarwal PK, Grossman HB, et al. Outcome of patients with bladder cancer with $\mathrm{pN}+$ disease after preoperative chemotherapy and radical cystectomy. Urology 2009;73:147-52.

9. Kassouf W, Spiess PE, Siefker-Radtke A, et al. Outcome and patterns of recurrence of nonbilharzial pure squamous cell carcinoma of the bladder: A contemporary review of the university of texas $M D$ anderson cancer center experience. Cancer 2007;110:764-9.

10. Spiess PE, Kassouf W, Steinberg JR, et al. Review of the M.D. Anderson experience in the treatment of bladder sarcoma. Urol Oncol 2007;25:38-45.

11. Reuter VE. The pathology of bladder cancer. Urology 2006;67:11-7.

12. Tavora F, Epstein Jl. Bladder cancer, pathological classification and staging. BJU Int 2008;102:1216-20.

13. Wasco MJ, Daignault S, Zhang Y, et al. Urothelial carcinoma with divergent histologic differentiation (mixed histologic features) predicts the presence of locally advanced bladder cancer when detected at transurethral resection. Urology 2007;70:69-74.

14. Antunes AA, Nesrallah LJ, Dall' 0 glio MF, et al. The role of squamous differentiation in patients with transitional cell carcinoma of the bladder treated with radical cystectomy. Int Braz J Urol 2007;33:33945; discussion 346

15. Yang $M H$, Yen $C C$, Chen PM, et al. Prognostic-factors-based risk-stratification model for invasive urothelial carcinoma of the urinary bladder in taiwan. Urology 2002;59:232-8; discussion 238-9.

16. Lopez-Beltran A, Requena MJ, Alvarez-Kindelan J, et al. Squamous differentiation in primary urothelial carcinoma of the urinary tract as seen by MAC387 immunohistochemistry. J Clin Pathol 2007;60:332-5.

17. Erdemir F, Tunc $M, O z c a n F$, et al. The effect of squamous and/or glandular differentiation on recurrence, progression and survival in urothelial carcinoma of bladder. Int Urol Nephrol 2007;39:803-7.

18. Honma I, Masumori N, Sato E, et al. Local recurrence after radical cystectomy for invasive bladder cancer: An analysis of predictive factors. Urology 2004;64:744-8.

19. Martin JE, Jenkins BJ, Zuk RJ, et al. Clinical importance of squamous metaplasia in invasive transitional cell carcinoma of the bladder. J Clin Pathol 1989;42:250-3.

20. Logothetis $\mathrm{CJ}$, Johnson DE, Chong C, et al. Adjuvant cyclophosphamide, doxorubicin, and cisplatin chemotherapy for bladder cancer: An update. J Clin Oncol 1988;6:1590-6.

21. Holmang $S$, Johansson SL. The nested variant of transitional cell carcinoma-a rare neoplasm with poor prognosis. Scand I Urol Nephrol 2001;35:102-5.

22. Drew PA, Furman J, Civantos F, et al. The nested variant of transitional cell carcinoma: An aggressive neoplasm with innocuous histology. Mod Pathol 1996;9:989-94.

23. Alvarado-Cabrero I, Sierra-Santiesteban Fl, Mantilla-Morales A, et al. Micropapillary carcinoma of the urothelial tract. A clinicopathologic study of 38 cases. Ann Diagn Pathol 2005;9:1-5.

24. Amin MB, Ro JY, el-Sharkawy T, et al. Micropapillary variant of transitional cell carcinoma of the urinary bladder. histologic pattern resembling ovarian papillary serous carcinoma. Am J Surg Pathol 1994;18: 1224-32.

25. Trabelsi A, Stita W, Soumaya R, et al. Micropapillary carcinoma of the urinary bladder: A case report and review of the literature. Can Urol Assoc J 2008;2:540-2.

26. Johansson SL, Borghede G, Holmang S. Micropapillary bladder carcinoma: A clinicopathological study of 20 cases. J Urol 1999;161:1798-802.

27. Samaratunga $\mathrm{H}$, Khoo K. Micropapillary variant of urothelial carcinoma of the urinary bladder; a clinicopathological and immunohistochemical study. Histopathology 2004:45:55-64.

28. Kamat AM, Dinney CP, Gee JR, et al. Micropapillary bladder cancer: A review of the university of texas M. D. Anderson cancer center experience with 100 consecutive patients. Cancer 2007;110:62-7.

29. Kamat AM, Gee JR, Dinney CP, et al. The case for early cystectomy in the treatment of nonmuscle invasive micropapillary bladder carcinoma. J Urol 2006;175:881-5.

30. Fadare 0 , Renshaw IL, Rubin C. Pleomorphic lymphoepithelioma-like carcinoma of the urinary bladder. Int J Clin Exp Pathol 2009;2:194-9.

31. Tamas EF, Nielsen ME, Schoenberg MP, et al. Lymphoepithelioma-like carcinoma of the urinary tract: A clinicopathological study of 30 pure and mixed cases. Mod Pathol 2007;20:828-834.

32. Chikwava KR, Gingrich JR, Parwani AV. Lymphoepithelioma-like carcinoma of the urinary bladder. Pathology 2008;40:310-1.

33. Lopez-Beltran A, Luque RJ, Vicioso L, et al. Lymphoepithelioma-like carcinoma of the urinary bladder: A clinicopathologic study of 13 cases. Virchows Arch 2001;438:552-7.

34. Amin MB, Ro JY, Lee KM, et al. Lymphoepithelioma-like carcinoma of the urinary bladder. Am I Surg Pathol 1994; 18:466-73.

35. Shanks JH, Iczkowski KA. Divergent differentiation in urothelial carcinoma and other bladder cancer subtypes with selected mimics. Histopathology 2009;54:885-900.

36. Fritsche HM, Burger $M$, Denzinger $S$, et al. Plasmacytoid urothelial carcinoma of the bladder: Histological and clinical features of 5 cases. J Urol 2008;180:1923-7.

37. Mai KT, Park PC, Yazdi HM, et al. Plasmacytoid urothelial carcinoma of the urinary bladder report of seven new cases. Eur Urol 2006;50:1111-4.

38. Lopez-Beltran A, Pacelli A, Rothenberg HJ, et al. Carcinosarcoma and sarcomatoid carcinoma of the bladder: Clinicopathological study of 41 cases. J Urol 1998;159:1497-503. 
Chalasani et al.

39. Sung MT, Wang M, Maclennan GT, et al. Histogenesis of sarcomatoid urothelial carcinoma of the urinary bladder: Evidence for a common clonal origin with divergent differentiation. J Pathol 2007;211:420-30.

40. Armstrong AB, Wang M, Eble JN, et al. TP53 mutational analysis supports monoclonal origin of biphasic sarcomatoid urothelial carcinoma (carcinosarcoma) of the urinary bladder. Mod Pathol 2009;22:113-8.

41. Black PC, Brown GA, Dinney CP. The impact of variant histology on the outcome of bladder cancer treated with curative intent. Urol Oncol 2009;27:3-7.

42. Wright IL, Black $\mathrm{PC}$, Brown $\mathrm{GA}$, et al. Differences in survival among patients with sarcomatoid carcinoma, carcinosarcoma and urothelial carcinoma of the bladder. J Urol 2007;178: 2302-6; discussion 2307.

43. Black PC, Brown GA, Dinney CP. Clinical and therapeutic significance of aberrant differentiation patterns in bladder cancer. Expert Rev Anticancer Ther 2007;7:1015-26.

44. Neoadjuvant cisplatin, methotrexate, and vinblastine chemotherapy for muscle-invasive bladder cancer: A randomised controlled trial. international collaboration of trialists. Lancet 1999;354:533-40.

45. De Santis M, Bachner M. New developments in first- and second-line chemotherapy for transitional cell, squamous cell and adenocarcinoma of the bladder. Curr Opin Urol 2007;17:363-8.

46. Black PC, Brown GA, Grossman HB, et al. Neoadjuvant chemotherapy for bladder cancer. World I Urol 2006;24:531-42

47. Rogers CG, Palapattu GS, Shariat SF, et al. Clinical outcomes following radical cystectomy for primary nontransitional cell carcinoma of the bladder compared to transitional cell carcinoma of the bladder. J Urol 2006; 175:2048-53; discussion 2053.

48. Ghoneim MA, et-Mekresh MM, el-Baz MA, et al. Radical cystectomy for carcinoma of the bladder: Critical evaluation of the results in 1,026 cases. J Urol 1997;158:393-9.

49. El-Sebaie M, Zaghloul MS, Howard G, et al. Squamous cell carcinoma of the bilharzial and non-bilharzial urinary bladder: A review of etiological features, natural history, and management. Int J Clin Oncol 2005; 10:20-5.

50. Scosyrev E, Yao J, Messing E. Urothelial carcinoma versus squamous cell carcinoma of bladder: Is survival different with stage adjustment? Urology 2009;73:822-7.

51. Lagwinski N, Thomas A, Stephenson AJ, et al. Squamous cell carcinoma of the bladder: A clinicopathologic analysis of 45 cases. Am J Surg Pathol 2007;31:1777-87.

52. Zaghloul MS, Awwad HK, Akoush HH, et al. Postoperative radiotherapy of carcinoma in bilharzial bladder: Improved disease free survival through improving local control. Int J Radiat Oncol Biol Phys 1992;23: $511-7$.
53. Gad el Mawla N, Mansour MA, Eissa S, et al. A randomized pilot study of high-dose epirubicin as neoadjuvant chemotherapy in the treatment of cancer of the bilharzial bladder. Ann Oncol 1991;2:137-40.

54. Abol-Enein H, Kava BR, Carmack AJ. Nonurothelial cancer of the bladder. Urology 2007;69:93-104.

55. Lynch CF, Cohen MB. Urinary system. Cancer 1995;75:316-29.

56. Thomas AA, Stephenson AJ, Campbell SC, et al. Clinicopathologic features and utility of immunohistochemical markers in signet-ring cell adenocarcinoma of the bladder. Hum Pathol 2009;40:108-16.

57. Wright JL, Porter MP, Li Cl, et al. Differences in survival among patients with urachal and nonurachal adenocarcinomas of the bladder. Cancer 2006;107:721-8.

58. el-Mekresh MM, el-Baz MA, Abol-Enein $\mathrm{H}$, et al. Primary adenocarcinoma of the urinary bladder: A report of 185 cases. Br J Urol 1998;82:206-12.

59. Zaghloul MS, Nouh A, Nazmy M, et al. Long-term results of primary adenocarcinoma of the urinary bladder: A report on 192 patients. Urol Oncol 2006;24:13-20.

60. Siefker-Radtke A. Urachal carcinoma: Surgical and chemotherapeutic options. Expert Rev Anticancer Ther 2006;6:1715-21.

61. Alijo Serrano F, Sanchez-Mora N, Angel Arranz J, et al. Large cell and small cell neuroendocrine bladder carcinoma: Immunohistochemical and outcome study in a single institution. Am I Clin Pathol 2007;128:733-9.

62. Siefker-Radtke A0, Dinney CP, Abrahams NA, et al. Evidence supporting preoperative chemotherapy for small cell carcinoma of the bladder: A retrospective review of the M. D. Anderson cancer experience. J Urol 2004; 172:481-4.

63. Mukesh M, Cook N, Hollingdale AE, et al. Small cell carcinoma of the urinary bladder: A 15-year retrospective review of treatment and survival in the Anglian Cancer Network. BJU Int 2009; 103:747-52.

64. Bex A, de Vries R, Pos F, et al. Long-term survival after sequential chemoradiation for limited disease small cell carcinoma of the bladder. World I Urol 2009;27:101-6.

65. Tsili AC, Giannakis D, Sofikitis N, et al. Small cell carcinoma of the urinary bladder: Virtual CT cystoscopic findings. J Postgrad Med 2009;55:33-4.

66. Mackey JR, Au HJ, Hugh J, et al. Genitourinary small cell carcinoma: Determination of clinical and therapeutic factors associated with survival. J Urol 1998;159:1624-9.

Correspondence: Dr. Jonathan I. Izawa, Associate Professor, Departments of Surgery \& Oncology, Divisions of Urology \& Surgical Oncology, London Health Sciences Centre-Victoria Hospital, 800 Commissioners Road East, London, ON N6A 465; fax: 519-685-8450; jonathan.izawa@lhsc.on.ca 